\title{
Diffraction of atoms from stepped surfaces: A semiclassical chaotic $S$-matrix study
}

\author{
R. Guantes, F. Borondo, and Charles Jaffé* \\ Departamento de Química, C-IX, Universidad Autónoma de Madrid, Cantoblanco, 28049 Madrid, Spain
}

\author{
S. Miret-Artés \\ Instituto de Matemáticas y Física Fundamental, Consejo Superior de Investigaciones Científicas, Serrano 123, 28006 Madrid, Spain
}

(Received 11 April 1995; revised manuscript received 4 December 1995)

\begin{abstract}
The diffraction of atoms from stepped surfaces is treated using the semiclassical $S$-matrix theory. Two different regimes of the behavior of the classical trajectories, regular and chaotic, are considered. For the latter case, the diffraction order function presents a fractal structure, and there is an infinite number of trajectories connecting the initial and final states. Using the scaling laws of the fractal, within the formalism of the $S$-matrix theory, we have obtained diffraction and specular intensities, which are in fairly good agreement with the experimental and quantum close-coupling results. [S0163-1829(96)00221-4]
\end{abstract}

\section{INTRODUCTION}

Presently, it is well accepted that diffraction patterns arising in atom-surface scattering are exclusively the result of the interference of each incident particle with itself. ${ }^{1}$ Consequently, this process can be best treated within a quantummechanical formalism where the superposition principle holds. However the semiclassical $S$-matrix theory, as developed independently in the early 1970's by Marcus and Miller, ${ }^{2}$ represents an alternative route, since it combines classical mechanics with this quantum principle. The main advantage of this semiclassical analysis is the physical insight gained since the dynamics of the process is explicitly included.

The validity of semiclassical $S$-matrix theory for elastic atom-surface scattering was tested in the past by Doll, ${ }^{3 \mathrm{a}} \mathrm{Mc}$ Cann and Celli, ${ }^{3 \mathrm{~b}}$ and Miller and co-workers, ${ }^{4}$ and comparison with other methods showed ${ }^{4 a}$ that the semiclassical $S$-matrix calculations worked even better than other approximate quantum methods. However the semiclassical theory has several shortcomings that we want to discuss and address in this paper.

The semiclassical theory can be viewed, in a very precise sense, as the stationary phase approximation (SPA) to quantum mechanics. The SPA is valid whenever $\hbar$ is small with respect to the total action of the system, and breaks down when neighboring trajectories in coordinate space encounter a caustic. This usually occurs in the vicinity of a classical turning point, and then Maslov's theory ${ }^{5}$ should be used, which allows us to uniformize the semiclassical approximation. These are limits inherent to the SPA.

Another failure arises when the SPA is applied to find the semiclassical limit of the $S$-matrix in scattering problems. The $S$-matrix elements are constructed using all the classical trajectories connecting the desired initial and final states. If two or more of the contributing trajectories give final states (as a function of the initial states) that are close together, then the amplitude for the $S$-matrix element diverges. This is related to the well-known rainbow effect in scattering theory. In this case catastrophe theory ${ }^{6}$ can be applied to obtain the appropriate uniform approximations. In the early stages of the semiclassical theory, Connor, Marcus, and Miller ${ }^{7}$ developed uniform approximations and showed how these approximations reduce to the usual primitive semiclassical formula when the trajectories are far enough from the catastrophe. Berry ${ }^{6 a}$ studied the case of cusped rainbows in scattering of atoms from a rippling-mirror surface.

Another problem which does not stem from a breakdown of the classical $S$-matrix theory but is entirely due to the extreme complexity of the dynamics of the system arises when we have irregular or chaotic scattering. ${ }^{8}$ In this case there exist an infinite number of classical trajectories connecting the initial and final states. This was observed by Rankin and Miller for a gas-phase chemical reaction, ${ }^{9}$ and they proposed to divide the $S$-matrix sum over trajectories in two terms: a contribution from direct trajectories (only a finite number), plus a contribution coming from the complex trajectories (a countable infinity), and computed statistically this last part (random phase approximation). Other authors have also considered this problem. ${ }^{10-13}$ The approaches range from just summing up a few contributions of complex forming trajectories, such as in the work of Stine and Marcus, ${ }^{10}$ to the use of some scaling properties to compute contributions to the scattering cross sections in the more recent work of Refs. $12 \mathrm{~b}$ and $13 \mathrm{~b}$.

The chaotic region, also called the "chattering" region, corresponding to complex forming trajectories (trapped trajectories in the case of atom-surface scattering), presents a fractal structure. This structure appears in all chaotic scattering problems. ${ }^{14-17}$ In a recent paper, Tiyapan and Jaffé ${ }^{13 a}$ have studied the classical dynamics of collisional complexes, formed in gas-phase $\mathrm{He}-\mathrm{I}_{2}$ scattering, and made clear the self-similarity of the fractal structure. From initial phase angle-final action plots, they found the scaling laws, as well as the fractal dimension of the Cantor set. More recently, a classical study ${ }^{17}$ of the elastic scattering of the ${ }^{4} \mathrm{He}$ from the highly corrugated and stepped $\mathrm{Cu}(117)$ surface has demonstrated that the dynamics are chaotic for certain values of the incident angle and scattering energy of the incoming atoms. The structure of the corresponding fractal has been analyzed in detail, ${ }^{17 \mathrm{~b}}$ and we have also demonstrated that the onset of chaos is intimately related to classical surface rainbows. The 
interest of this system stems from the fact that it has been extensively studied experimentally ${ }^{18}$ and close-coupling results ${ }^{19}$ are available, and also a variety of other phenomena apart from diffraction, such as surface rainbows and selective adsorption resonances, occur.

In this paper we extend the work reported in Ref. 17 by presenting a quantitative analysis of the chaotic scattering processes taking place in the diffraction of atoms from very corrugated surfaces, $\mathrm{Cu}(115)$ and $\mathrm{Cu}(117)$, within the semiclassical formalism. A comparison between experimental and theoretical (semiclassical and close-coupling) results in a chaotic regime is presented.

The organization of the paper is as follows. In Sec. II we will briefly describe the system and the semiclassical $S$-matrix theory for atom-surface scattering. Results for a regular case will be presented, which can be taken as an illustration of the theory and also constitutes a test case of the semiclassical calculation method for the case of atomstepped surface scattering. Section III reviews the analysis of the fractal structure appearing in the classical chaotic regime, and the scaling laws and fractal dimension are calculated. The consequences of this structure for the classical $S$-matrix theory are discussed, and results of diffraction intensities for different initial energies and incident angles are presented. These results are compared with quantum close-coupling and experimental results. Finally, we discuss the difficulties involved in our treatment and future developments.

\section{SYSTEM DESCRIPTION AND SEMICLASSICAL $S$-MATRIX CALCULATIONS}

The elastic collisions of ${ }^{4} \mathrm{He}$ with two stepped $\mathrm{Cu}$ surfaces with an increasing degree of corrugation, (115) and (117), are studied in this paper. The corrugation of these surfaces lies primarily in the direction perpendicular to the

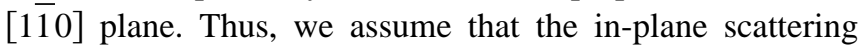
predominates. This is confirmed experimentally for the (115) surface and to a lesser degree for the (117) surface.

Consequently, we can model this system with the twodegrees-of-freedom Hamiltonian:

$$
H\left(P_{x}, P_{z}, x, z\right)=\frac{P_{x}^{2}+P_{z}^{2}}{2 m}+V(x, z),
$$

where $x$ and $z$ are, respectively, the parallel and perpendicular distances of the He particle to the $\mathrm{Cu}$ surface, and $V(x, z)$ a corrugated Morse function whose parameters have been taken from the literature. ${ }^{18 a, 19}$ Note that the zero of the energy scale is defined as the He at infinite separation and at rest.

Classical trajectories are calculated using the following initial conditions for the Hamiltonian's equations of motion:

$$
\begin{gathered}
z_{0}=z_{\max }, \\
x_{0}=-z_{\max } \tan \theta_{i}+b a, \\
P_{z_{0}}=-\sqrt{2 m E} \cos \theta_{i}, \\
P_{x_{0}}=\sqrt{2 m E} \sin \theta_{i},
\end{gathered}
$$

where $z_{\max }$ represents a value of $z$ sufficiently large that the coupling potential can be neglected, $b$ is the normalized im- pact parameter $(0 \leqslant b \leqslant 1)$, and $\theta_{i}$ the initial incident angle. All trajectories are integrated until the $\mathrm{He}$ is once again in the asymptotic region $\left(z>z_{\max }\right)$ with sufficient energy to escape from the surface $\left[H_{z}\left(P_{z}, z\right)>0\right]$, when all final values of various magnitudes are calculated. All calculations reported here are for a collision energy of $21 \mathrm{meV}$.

The diffraction intensities are constructed from the $S$-matrix elements:

$$
I_{J}(E)=\left|S_{J}(E)\right|^{2}
$$

where $J$ is an integer labeling the diffraction channel. The semiclassical approximation to the $S$ matrix is given as a sum over trajectories:

$$
S_{J}(E)=\sum_{n}\left|\frac{\partial J}{\partial b}\right|_{b=b_{n}}^{-1 / 2} \exp \left[i\left(\frac{\Phi_{n}}{\hbar}-\frac{\pi \mu_{n}}{2}\right)\right] .
$$

The diffraction order function $J(b)$ is defined by

$$
J(b)=\frac{\left(P_{x_{f}}-P_{x_{0}}\right) a}{2 \pi \hbar},
$$

the classical action $\Phi$ by

$$
\Phi\left(P_{x_{0}}, P_{x_{f}}\right)=-\int_{t_{0}}^{t_{f}} d t\left(x \dot{P}_{x}+z \dot{P}_{z}\right),
$$

and $\mu$ is the Maslov index. ${ }^{5}$

The sum in Eq. (4) is over all classical trajectories satisfying the diffraction condition: this requires that $J(b)$ be an integer. The pre-exponential factors and the classical actions in the terms of the sum (4) are evaluated at these points. Clearly, all the information required for the evaluation of this sum can be obtained in the construction of the $J$ vs $b$ plots.

Shown in Fig. 1 is the diffraction order function $J(b)$ as a function of the impact parameter $b$ for two cases where only regular or direct scattering occurs: the dashed line is for the (115) surface of $\mathrm{Cu}$ and initial scattering angle of $32^{\circ}$, and the solid one for the (117) surface and initial angle of $36^{\circ}$. As seen here the diffraction order function is a smooth and well defined function. Consider first the (115) surface: classical trajectories satisfying the Bragg condition occur for $J=-5$, $-4, \ldots, 3$. At each of these values there are two contributing trajectories.

Observe that for the $J=-5$ and $J=3$ diffraction channels the two contributing trajectories are close together. In these circumstances the primitive semiclassical approximation is expected to fail and one must use an uniformized approximation, ${ }^{2}$ given in this case by

$$
I_{J}(E)=\pi \sqrt{y^{\prime}}\left[\left(p_{1}+p_{2}\right)^{2} A i^{2}\left(-y^{\prime}\right)+\left(p_{1}-p_{2}\right)^{2} B i^{2}\left(-y^{\prime}\right)\right],
$$

where $p_{1}$ and $p_{2}$ are the pre-exponential factors defined in Eq. (4), $A i\left(-y^{\prime}\right)$ and $B i\left(-y^{\prime}\right)$ are the Airy functions of first and second kind, ${ }^{20}$ and $y^{\prime}=\left[(3 / 4)\left(\Phi_{1}-\Phi_{2}\right) / \hbar\right]^{2 / 3}$.

Transitions to the $J=-6$ and -7 diffraction channels are classically forbidden but energetically allowed, since they are observed experimentally. The contribution from classically forbidden processes is accomplished by analytical continuation $^{21}$ of the diffraction order function at the rainbow angle to find complex solutions satisfying the Bragg condition. In the present case $J(b)$ was fitted to a quartic 


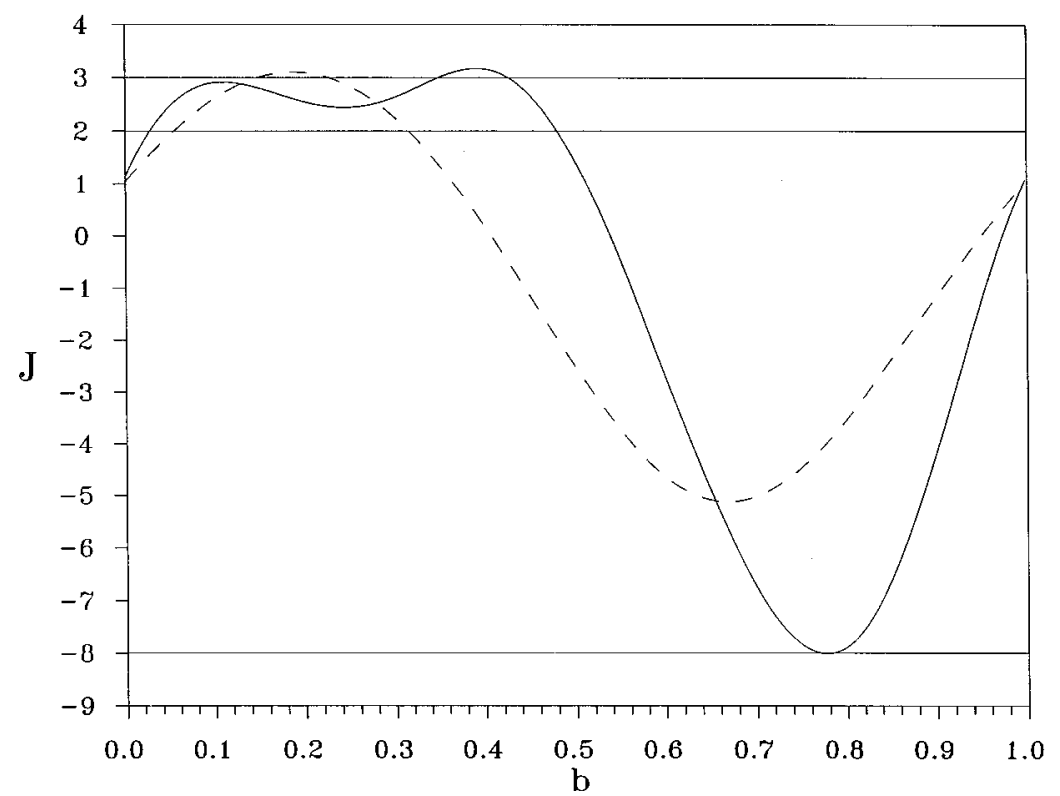

FIG. 1. Diffraction order function $J(b)$ vs impact parameter $b$ for the scattering of ${ }^{4} \mathrm{He}$ atoms at a total energy of $21 \mathrm{meV}$ from two different surfaces of $\mathrm{Cu}$ : a $\mathrm{Cu}(115)$ surface at incident angle $\theta_{i}=32^{\circ}$ (dashed line) and a $\mathrm{Cu}(117)$ surface for an incident angle $\theta_{i}=36^{\circ}$ (solid line). To guide the eye we have included the lines corresponding to channels where either a uniform approximation is needed or the contribution from forbidden trajectories are expected to be important (see text). function in the neighborhood of the rainbow angle, and the complex roots were found. Then the corresponding classically forbidden trajectories were calculated in complexvalued phase space. The primitive and uniform semiclassical approximations for the diffraction intensity becomes in this case

$$
\begin{gathered}
I_{J}(E)=p^{2} \exp \left(-2 \operatorname{Im} \frac{\Phi}{\hbar}\right) \quad(\text { primitive }), \\
I_{J}(E)=4 p^{2} \pi y^{1 / 2} A i^{2}(y) \quad \text { (uniform) }
\end{gathered}
$$

where $y=[(3 / 2) \operatorname{Im}(\Phi / \hbar)]^{2 / 3}, p=(\partial J / \partial b)^{1 / 2}$, and $\Phi$ is the classical action of the classically forbidden trajectory.

The diffraction intensities for He scattering from the (115) surface of $\mathrm{Cu}$ are given in Table I. They are compared with our close-coupling calculations and experimental results (note that the in-plane experimental results are normalized to unity ${ }^{18}$ ). We see that the agreement between the semiclassical and close-coupling results is excellent for both the classically allowed and the classically forbidden transitions.

TABLE I. Diffraction intensities for the scattering of ${ }^{4} \mathrm{He}$ atoms from a $\mathrm{Cu}(115)$ surface at $21 \mathrm{meV}$ and $\theta_{i}=32^{\circ}$.

\begin{tabular}{rccc}
\hline \hline $\begin{array}{c}\text { Diffraction } \\
\text { channel }\end{array}$ & Semiclassical & Close-coupling & $\begin{array}{c}\text { Expt. } \\
\text { (Ref. 18) }\end{array}$ \\
\hline 3 & 0.137 & 0.148 & 0.125 \\
2 & 0.228 & 0.219 & 0.234 \\
1 & 0.0056 & 0.0037 & \\
0 & 0.149 & 0.152 & 0.190 \\
-1 & 0.059 & 0.059 & 0.060 \\
-2 & 0.022 & 0.022 & 0.019 \\
-3 & 0.148 & 0.148 & 0.116 \\
-4 & 0.149 & 0.149 & 0.153 \\
-5 & 0.073 & 0.072 & 0.099 \\
$-6^{\mathrm{a}}$ & 0.023 & 0.022 & \\
$-7^{\mathrm{a}}$ & 0.0062 & 0.0039 & \\
\hline \hline
\end{tabular}

${ }^{\mathrm{a}}$ Classically forbidden transitions.
Next we consider the scattering from the (117) surface of $\mathrm{Cu}$ (solid line in Fig. 1). This surface is more highly corrugated and more diffraction channels are open. As a consequence, the intensity in the specular channel decreases in favor of the higher-order diffraction channels. In general, the interaction between the He atom and the surface is stronger and the experimentally observed diffraction pattern has more oscillations. In the present case there are four rainbow angles. Transitions to the diffraction channels labeled by $J=$ $-8,-7, \ldots, 3$ are classically allowed, while transitions to the $J \leqslant-9$ diffraction channels are energetically allowed but classically forbidden. For the two diffraction channels labelled $J=2$ and $J=3$ we expect important contributions to the transition probabilities from the classically forbidden processes associated with the rainbows in addition to the classically allowed contributions. The semiclassical diffraction intensities are compared with the close-coupling results in Table II. The semiclassical results given in the second column were obtained by simply adding the contributions from the classically allowed and classically forbidden trajectories, that is, the interference between these two processes has been neglected. The difference with the close-coupling results is noticeable for the channels $J=2$ and $J=3$. Moreover, when adding all calculated intensities, an important loss of unitarity is found. This interference has been included in the results presented in the third column. The contribution from this interference is given by

$$
\begin{aligned}
4 \pi q p\left[\left(p_{1}+p_{2}\right) A i\left(-y^{\prime}\right) A i(y) \cos \theta\right. \\
\left.+\left(p_{1}-p_{2}\right) B i\left(-y^{\prime}\right) A i(y) \sin \theta\right],
\end{aligned}
$$

where $q=\left(y y^{\prime}\right)^{1 / 4}$ and $\theta=\left(\Phi_{1}-\Phi_{2}\right) / 2 \hbar-\operatorname{Re}(\Phi+\pi / 4)$. Now, the agreement of the semiclassical results with the closecoupling results is much closer, and the unitarity is fully achieved. To the best of our knowledge, the importance of the interference terms between the classically allowed and the classically forbidden has not been widely recognized.

To this point in the discussion we have not addressed the issue of the Maslov's index. This number is an integer and is related to the topology of the Lagrangian manifold on which 
TABLE II. Diffraction intensities for the scattering of ${ }^{4} \mathrm{He}$ atoms from a $\mathrm{Cu}(117)$ surface at $21 \mathrm{meV}$ and $\theta_{i}=36^{\circ}$.

\begin{tabular}{rcccc}
\hline \hline $\begin{array}{c}\text { Diffraction } \\
\text { channel }\end{array}$ & $\begin{array}{c}\text { Semiclassical } \\
\text { (a) }\end{array}$ & $\begin{array}{c}\text { Semiclassical } \\
\text { (b) }\end{array}$ & $\begin{array}{c}\text { Close- } \\
\text { coupling }\end{array}$ & $\begin{array}{c}\text { Expt. } \\
\text { (Ref. 18) }\end{array}$ \\
\hline 3 & 0.163 & 0.227 & 0.29 & 0.769 \\
2 & 0.153 & 0.277 & 0.229 & 0.166 \\
1 & 0.063 & 0.063 & 0.019 & \\
0 & 0.0048 & 0.0048 & 0.0077 & \\
-1 & 0.072 & 0.072 & 0.088 & 0.051 \\
-2 & 0.057 & 0.057 & 0.061 & 0.013 \\
-3 & 0.0037 & 0.0037 & 0.0043 & \\
-4 & 0.020 & 0.020 & 0.019 & \\
-5 & 0.071 & 0.071 & 0.070 & \\
-6 & 0.089 & 0.089 & 0.088 & \\
-7 & 0.068 & 0.068 & 0.066 & \\
-8 & 0.043 & 0.043 & 0.036 & \\
-9 & 0.019 & 0.019 & 0.021 & \\
\hline \hline
\end{tabular}

${ }^{\text {a }}$ Results including the contribution from the direct scattering plus classically forbidden transitions for channels $J=2$ and 3 which are close to the rainbows (see Fig. 2).

${ }^{b}$ Same as (a) but including the interference terms between classically allowed and classically forbidden trajectories.

${ }^{\mathrm{c}}$ Classically forbidden transitions.

the trajectory is confined. Each time the trajectory encounters a caustic the semiclassical approximation suffers a singularity. The semiclassical approximation on both sides of the caustic can be joined by using Maslov's theory. ${ }^{5}$ In this manner one discovers that at each caustic one must add a phase to the primitive semiclassical results. This phase is given by the Maslov's index. In the present example there are two types of caustics when we project the Lagrangian manifold on the coordinate plane. These caustics are shown in Fig. 2. Trajectories at different sides of a rainbow angle differ in the crossing of one caustic, which has the typical form of a cusp. However, we numerically determined that the encounter with this caustic do not cause additional phase change. Thus, all trajectories contributing to a given $S$-matrix element have the same Maslov's index. Furthermore, since only differences of the classical actions occur in the expressions for the diffraction intensities, Maslov's index plays no role at this level of the theory.

In summary, we conclude that semiclassical $S$-matrix theory as applied in this section yields diffraction intensities that are in good agreement with close-coupling results for the scattering of atoms from stepped surfaces where the dynamics are regular, and the diffraction order function is a smooth, well-behaved function. In the next section the extension of the classical $S$-matrix theory to the case that the diffraction order function is an ill-behaved function and the classical dynamics are chaotic will be discussed.

\section{FRACTAL STRUCTURES AND SCALING LAWS}

Shown in Fig. 3 are the diffraction order functions for the scattering of $\mathrm{He}$ from the (117) surface of $\mathrm{Cu}$ at incident angles of $45^{\circ}$ and $75^{\circ}$. Here, in contrast to the examples seen in the previous section, $J(b)$ is not a smooth, well-behaved function. Rather, it shows ill-behaved chattering regions

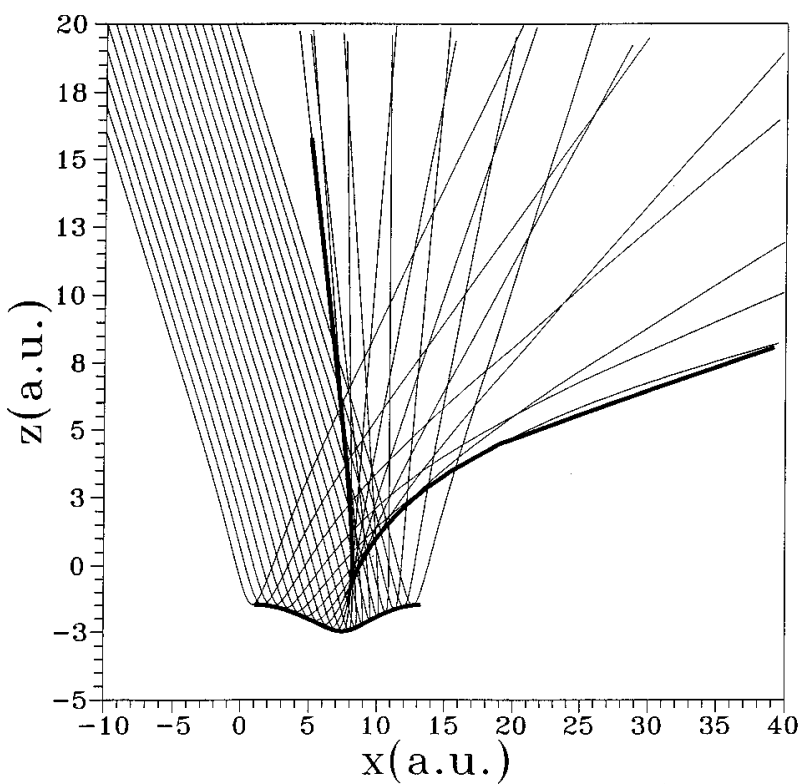

FIG. 2. Projection on the coordinates plane of a series of trajectories corresponding to different values of the impact parameter, $b$, for the scattering from the $\mathrm{Cu}(115)$ surface at a total energy of 21 $\mathrm{meV}$ and $\theta_{i}=32^{\circ}$. At the first turning point the pre-exponential factor of the WKB wave function is singular, and the projection of these singular points form a caustic (thick solid line). Trajectories starting at different sides of the first rainbow in Fig. 1 also encounters a second caustic.

where, at first sight, the trajectories appear to leave the surface with random values of $J$. The evidence that the chattering region was not completely random or statistical was presented by Gottdiener for the $\mathrm{H}+\mathrm{H}_{2}$ reaction. ${ }^{15}$ Subsequently, Noid, Gray, and Rice ${ }^{16}$ recognized the fractal character of the chattering regions and calculated covering dimension by box counting. More recently, Tiyapan and Jaffé $^{13}$ have analyzed the structure of these fractals in greater detail. They have demonstrated the asymptotic self-similarity of the chattering region and have determined the scaling parameters and the fractal dimension. In a subsequent paper they have considered the extension of classical $S$-matrix theory to chaotic scattering systems. In the present paper we will follow, with minor modification, the procedures discussed there and apply them to the present problem.

The application of classical $S$-matrix theory to systems that are chaotic is problematical. The major difficulty is that there are an infinite number of trajectories that contribute to the sum in Eq. (4). The manner in which to evaluate the sum is not obvious and requires a deep understanding of the structure of the chattering region. If we expand the chattering regions seen in Fig. 3, we observe that they consist of a series of smooth subdomains, called "icicles," separated by gaps of more irregular behavior. Subsequent expansions show that the same pattern can be seen at every scale, forming a hierarchy of different generations.

The positions of the icicles within the chattering region provide information concerning the dynamics of the system. In the present case, they correspond to trajectories for which a sufficient amount of energy has been transferred from the perpendicular to the parallel degree of freedom so that they are temporarily trapped in the attractive well close to the 

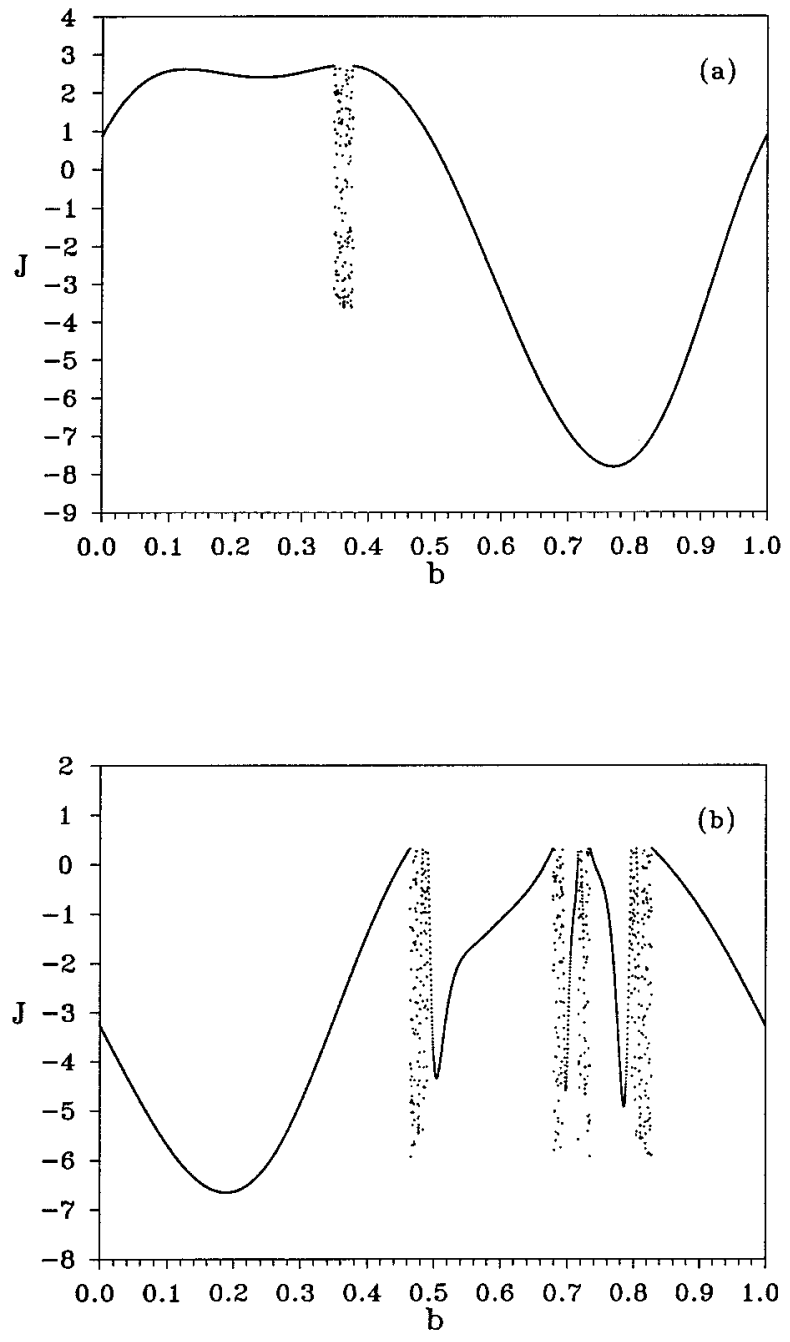

FIG. 3. (a) Diffraction order function $J(b)$ vs impact parameter $b$ for the scattering of ${ }^{4} \mathrm{He}$ atoms from a $\mathrm{Cu}(117)$ surface at a total energy of $21 \mathrm{meV}$ and for an incident angle $\theta_{i}=45^{\circ}$. Notice that the third rainbow has turned into a chattering region. (b) Same as (a) but for $\theta_{i}=75^{\circ}$. Now the three rainbows have reached the trapping conditions giving a much wider chattering region than in the previous case.

surface. All of the trajectories associated with a given icicle are characterized by the number of bounces with the surface and the number of unit cells traveled in each bounce. Then, we can use a symbolic labeling to classify the icicles. Icicles in the same generation have the same number of bounces (two for the first generation, three for the second, etc.) and they differ in the number of unit cells traveled in the last jump. A unique labeling scheme along these lines is discussed in detail in Ref. 17. In this scheme each icicle in the $n$th generation is labeled by $n$ signed integers. The values of the integers are equal to the number of unit cells accumulated up to each bounce and the sign of the number indicates whether the icicle appears to the right or left of the central feature in the previous generation. This labeling system will be used throughout this paper.

The self-similarity exhibited by the chattering regions can be understood with expansions of irregular gaps far enough from the center. In Fig. 4 we present the expansions of the irregular gaps between two consecutive pairs of first genera-
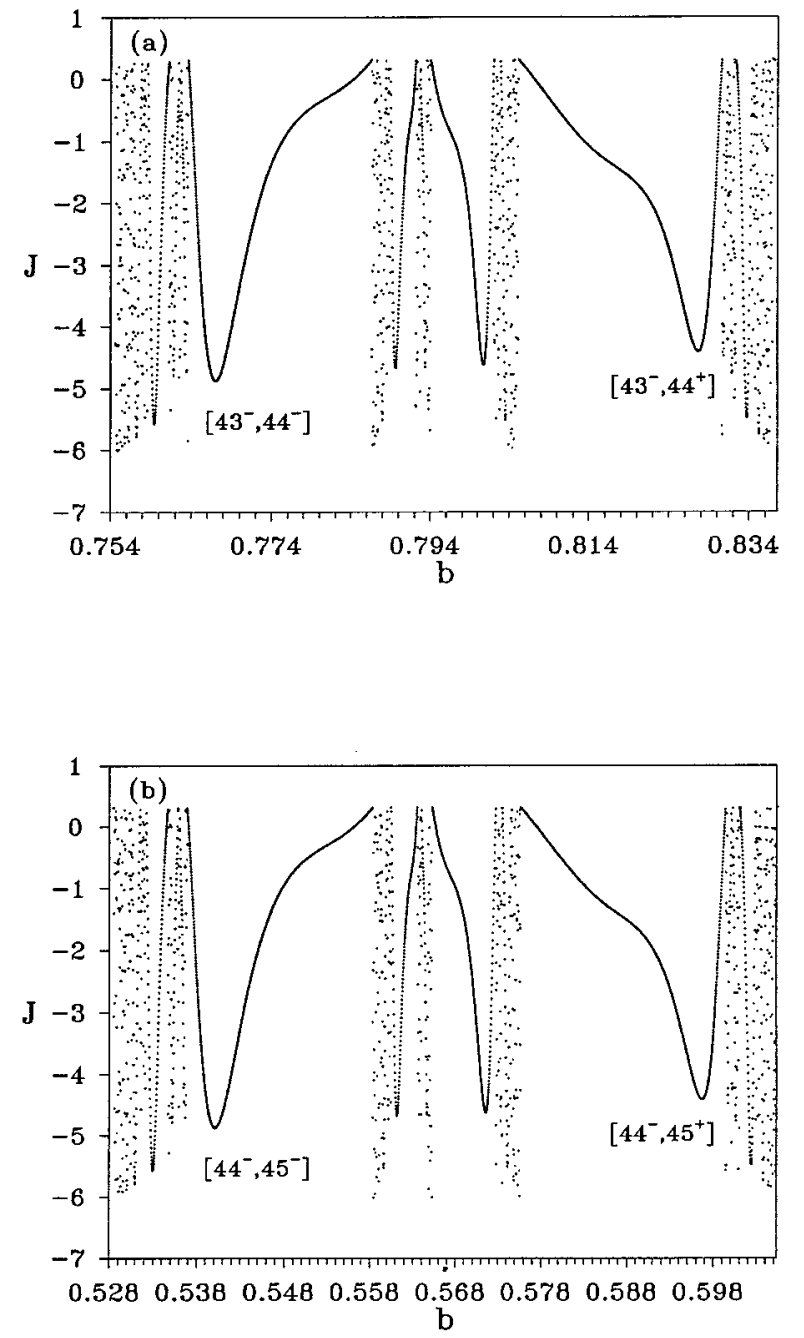

FIG. 4. Gaps between: (a) icicles $\left[42^{-}\right]$and $\left[43^{-}\right]$, and (b) icicles $\left[43^{-}\right]$and $\left[44^{-}\right]$, corresponding to the chattering region of Fig. 3(b), both drawn to the same scale. The (asymptotic) selfsimilarity of the fractal is evident.

tion icicles for an initial scattering angle of $75^{\circ}$. In Fig. 4(a) is shown the gap between the $\left[42^{-}\right]$and the $\left[43^{-}\right]$icicles and in Fig. 4(b) is shown the gap between the $\left[43^{-}\right]$and the $\left[44^{-}\right]$icicles. In our symbolic labeling scheme, these icicles correspond to trajectories that are trapped on the surface for a jump and travel 42,43 , and 44 unit cells, respectively, during the bounces. It is seen that both structures are virtually identical. This same result is observed for any pair of gaps between icicles of any generation provided that their position (family) within the generation is sufficiently high. In other words, the fractal is asymptotically self-similar. This implies that the icicles posses an invariant shape. This is illustrated in Fig. 5 where we have plotted superimposed images, scaled to unit height and width, of the $\left[10^{-}\right],\left[43^{-}\right]$, and the $\left[111^{-}\right]$icicles. The existence of an invariant shape of the icicles is quite clear. These results demonstrate the selfsimilarity within a given generation (intragenerational selfsimilarity).

The self-similarity between different generations of icicles can be demonstrated in the same manner, expanding the central gaps of consecutive generations, until we arrive at an invariant pattern. This suggests that, asymptotically, this 


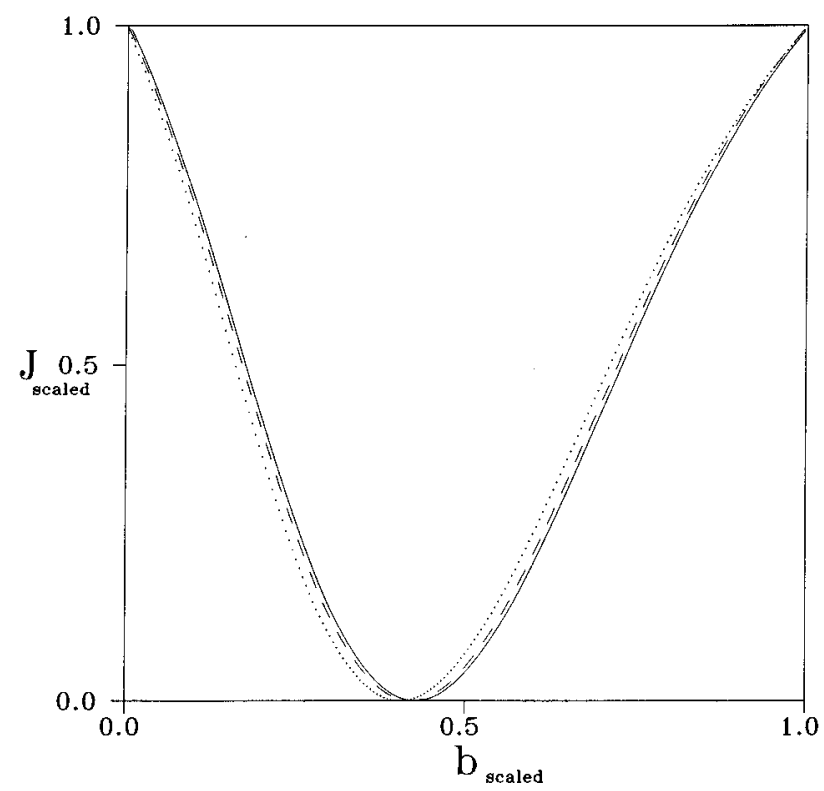

FIG. 5. Icicles $\left[10^{-}\right]$(dots), $\left[43^{-}\right]$(dashed line), and $\left[111^{-}\right]$ (solid line) corresponding to the chattering region of Fig. 3(b) scaled to height and width unity. The convergence of the form of the icicles to an invariant shape is clearly seen. This invariant shape is the same for the right part of the fractal, and for other incident angles.

fractal can be characterized by two scaling laws: ${ }^{13 a}$ the first one intragenerational $(\alpha)$ and the second intergenerational $(\beta)$. If one is able to determine the two scaling parameters then, in principle, the fractal can be reproduced (at least asymptotically) from the knowledge of a single icicle.

There are several possible ways of determining the values of the scaling parameters. For each scaling parameter we chose the most convenient.

We first consider the intragenerational scaling parameter. To obtain this parameter we compute the ratios of the widths of neighboring icicles in the first generation

$$
\alpha_{i}=\frac{W_{i+1}^{(1)}}{W_{i}^{(1)}}, \quad \text { for } i=1,2, \ldots, \infty .
$$

The value of this ratio in the limit $i \rightarrow \infty$ gives the value of the intragenerational scaling parameter. In the present case this was determined to be $\alpha_{\infty} \simeq 0.98$. Note that this value is valid for both the right- and left-hand sides of the fractal. The intragenerational scaling law is then given by

$$
W_{i}^{(1)}=\alpha_{\infty}^{i} W_{0}^{(1)},
$$

where $W_{0}^{(1)}$ is the width of the central icicle. Care must be taken when using this equation, as it is only valid in the asymptotic region. It should be noted that this scaling parameter can also be obtained by considering other quantities, for example, the height of the icicles, the positions of the icicles, the width of the gaps between icicles, etc. Further it is observed that the same value for this scaling parameter is found for subsequent generations.

In principle, the intergenerational scaling parameter $\beta$ can be found in a similar way. However it is very difficult to obtain it in this manner due to the loss of numerical precision as one approaches the asymptotic region. For this reason we have developed a different approach based on the relationship between the scaling parameters and the fractal dimension. Tiyapan and Jaffé ${ }^{13 a}$ have shown that

$$
\alpha^{d}+2 \beta^{d}=1
$$

where $d$ is the fractal dimension. They have used this relationship to calculate the fractal dimension. The approach taken here is to calculate first the fractal dimension and then use Eq. (12) to calculate the intergenerational scaling parameter.

The method that we use to calculate the fractal dimension is based on the existence of a Cantor set underlying the chattering region. This Cantor set is present in all chaotic scattering problems and include all of the trajectories that are homoclinic to the trajectory at infinite separation with the $\mathrm{He}$ atom traveling parallel to the surface. It will also include a variety of other trajectories including both hetero- and homoclinic orbits associated with various unstable periodic orbits. $^{22}$ The fractal dimension of this Cantor set in terms of the scaling parameters is given by Eq. (12) above.

The value of the fractal dimension can be determined using the well-known box-counting method. ${ }^{23}$ However this requires the use of an extremely large number of intervals as the size of the intervals becomes smaller and is numerically awkward. In the present case we have used an alternative procedure developed by Lau, Finn, and $\mathrm{Ott}^{24}$ for the calculation of the uncertainty dimension. The uncertainty dimension is equal to the box-counting dimension for typical dynamical systems and is much easier to compute. For our system, we implement this method as follows: We randomly choose an impact parameter $b$ within the chattering region. Then we run two trajectories whose initial conditions correspond to values of impact parameter $b$ and $b+\epsilon$, where $\epsilon$ is some small uncertainty. These two trajectories are followed until the collision is over. At this point it is determined whether or not the two trajectories are in the same icicle. If they are not, the pair of trajectories are said to be uncertain. We repeat this process, for the same value of the uncertainty, until we have found 100 uncertain pairs of trajectories and then calculate the fraction of uncertain trajectories $f(\epsilon)$. The fraction of uncertain trajectories is expected to scale exponentially with the fractal dimension, that is,

$$
d=1-\delta=1-\lim _{\epsilon \rightarrow 0} \frac{\ln f(\epsilon)}{\ln \epsilon} .
$$

Plotted in Fig. 6 is the logarithm of the fraction of uncertain trajectories $f(\boldsymbol{\epsilon})$ versus the logarithm of the uncertainty parameter $\epsilon$ for nine orders of magnitude. We observe that the uncertain fraction satisfies the relation $f(\epsilon) \simeq \epsilon^{\delta}$. The value of the fractal dimension is then determined from the value of the slope and is given by $d=0.63 \pm 0.01$. Using this value of the fractal dimension, the previously determined value of the intragenerational scaling parameter and Eq. (12), we determine the value of the intergenerational scaling parameter $\beta$ to be $0.0003 \pm 0.00005$. 


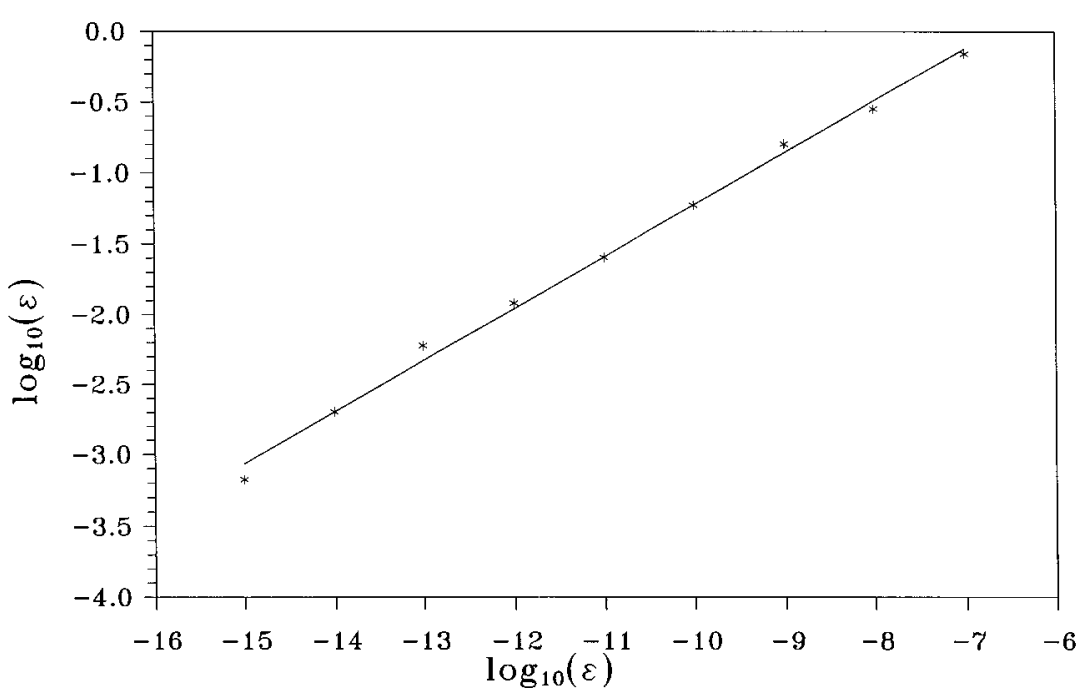

FIG. 6. Decimal logarithm of the uncertainty fraction vs the uncertainty. From the slope of the straight line the uncertainty dimension of the fractal Cantor set of singular points of the $J$ vs $b$ plots can be obtained. See text for details.
At this point we have characterized the asymptotic fractal properties of the chattering region. It is important to observe that these properties for a given crystallographic face are a function of the energy alone and are independent on the incident scattering angle. ${ }^{25}$ This result has important consequences from the computational point of view. It implies that the scaling parameters need only be determined once for each energy, and more importantly, can be determined using the most convenient incident angle. The invariance of the fractal properties with respect to the incident angle is related to the partition of phase space (actually the energy shell) into volumes associated with each of the topologically different families of trajectories. This partitioning represents an invariant fractal tiling of phase space $\mathrm{e}^{22}$ and can be constructed from the intersections of the stable and the unstable manifolds of the unstable trajectory at infinite separation and traveling parallel to the surface. Scattering at different incident angles explores different regions of this invariant fractal tiling, and thus, while the diffraction order function will have different structures, the underlying fractal properties of these structures are determined by the invariant fractal tiling. It can be demonstrated that the invariant shape of the icicles is identical to the shape of the direct region of the diffraction order function for an incident angle of $90^{\circ}$ and that the selfsimilar pattern observed in the asymptotic region is in fact identical to the chattering region for an incident angle of $90^{\circ}$. This discussion will be the subject of a future publication. ${ }^{25}$

The self-similarity exhibited by these fractals is crucial in the development of new analytical expressions for the $S$-matrix theory in the chaotic regime. We conclude this section with a discussion of the scaling properties of the quantities required to construct the contributions to the semiclassical $S$-matrix element from each contributing trajectory. These are the slopes of the diffraction order function and the classical actions for values of the impact parameter that satisfy the Bragg condition. Theoretical considerations ${ }^{13 a}$ lead one to expect that the slope and the difference of classical actions $\Phi_{1}-\Phi_{2}$ scale both as $\alpha$ for the intragenerational case and as $\beta$ for the intergenerational case. This has been confirmed numerically. The scaling of the contributions to the diffraction intensities is obtained by substituting the asymptotic expansions of the Airy functions ${ }^{20}$

$$
\begin{aligned}
& \pi y^{1 / 2} A i^{2}\left(-y^{\prime}\right) \sim \sin ^{2}\left(\frac{\pi}{4}+\frac{\Delta \Phi}{2 \hbar}\right), \\
& \pi y^{1 / 2} B i^{2}\left(-y^{\prime}\right) \sim \cos ^{2}\left(\frac{\pi}{4}+\frac{\Delta \Phi}{2 \hbar}\right),
\end{aligned}
$$

where $\Delta \Phi=\Phi_{2}-\Phi_{1}$, into Eq. (7) and expanding to first order. From this it is determined that the contributions to the diffraction intensities scale as $\alpha$ in the intragenerational case and as $\beta$ in the intergenerational case.

\section{SEMICLASSICAL $S$-MATRIX THEORY OF CHAOTIC ATOM-SURFACE SCATTERING}

As we stated before, for chaotic scattering problems the existence of an infinite number of trajectories connecting the initial and final states constitutes a great difficulty when trying to do the summation of Eq. (4). In an attempt, Rankin and Miller ${ }^{9}$ assumed that direct collisions and complex forming collisions contributed independently to the transition probability, and treated the complex part statistically. In this section we will check the feasibility of this assumption. Grayce, Skodje, and Hutson ${ }^{11}$ considered only specific families of complex orbits to account for the oscillations in chemical reaction probabilities. Very recently, Tiyapan and Jaffé $^{13 \mathrm{~b}}$ used the scaling laws of the fractal to extend the classical $S$-matrix theory for the $\mathrm{He}-\mathrm{I}_{2}$ collisions.

In the present treatment of the classical $S$-matrix theory applied to chaotic atom-surface scattering, the basic assumption is that the direct and complex contributions to the $S$ matrix (4) can be computed independently, and that inside the chattering region each icicle contributes independently to the scattering intensities, i.e., only the interference between trajectories corresponding to the same icicle is considered. This is equivalent to assume that the interferences between all trajectories in different icicles will cancel out on average. The diffraction intensities thus take the form

$$
I_{J}(E) \simeq I_{J}^{\text {direct }}+I_{J}^{\text {chattering }} \simeq\left|S_{J}^{\text {direct }}(E)\right|^{2}+\sum_{\text {icicles }}\left|S_{J}^{\text {icicle }}(E)\right|^{2} .
$$


The contribution from the direct trajectories is evaluated in the usual way (see Sec. II), and the contribution from the pair of trajectories in each icicle with the uniform classical $S$-matrix expression (7). However we still have a problem since the sum in Eq. (15) over icicles consists of an infinite number of elements. At this point we can take advantage of the fact that the individual probabilities obey asymptotic scaling laws, which can then be introduced in the second term of Eq. (15), giving a geometrical series which has a definite limit. For example, the contribution of the first generation, if we separate the central and the asymptotic parts is

$$
\begin{aligned}
I_{J}^{(1)}= & I_{J}^{[0]}(E)+\sum_{m=1}^{M-1}\left[I_{J}^{\left[m^{+}\right]}(E)+I_{J}^{\left[m^{-}\right]}(E)\right] \\
& +\sum_{m=M}^{\infty}\left[I_{J}^{\left[m^{+}\right]}(E)+I_{J}^{\left[m^{-}\right]}(E)\right],
\end{aligned}
$$

where the first term is the contribution of the central icicle, the second the contribution of the $M-1$ icicles in the regions where the scaling laws do not hold, and the third term is the asymptotic contribution of the fractal to the first generation. If we now make use of the scaling law (11), the third term in Eq. (16) becomes

$$
\begin{aligned}
\sum_{m=M}^{\infty}\left[I_{J}^{\left[m^{+}\right]}(E)+I_{J}^{\left[m^{-}\right]}(E)\right]= & \frac{1}{1-\alpha_{\infty}}\left[I_{J}^{\left[M^{+}\right]}(E)\right. \\
& \left.+I_{J}^{\left[M^{-}\right]}(E)\right] .
\end{aligned}
$$

Now making use of the intergenerational scaling parameter, the contribution of the second generation can then be expressed in terms of the contribution of the first generation as

$$
I_{J}^{(2)}(E)=2 \beta I_{J}^{(1)}(E) \sum_{i=1}^{\infty} \alpha_{\infty}^{i}=\frac{2 \beta \alpha_{\infty}}{1-\alpha_{\infty}} I_{J}^{(1)}(E),
$$

where the index $i$ runs over all of the gaps in the first generation. And, in general, the contribution from the $n$th generation is given by

$$
I_{J}^{(n)}(E)=\left[\frac{2 \beta \alpha_{\infty}}{1-\alpha_{\infty}}\right]^{n-1} I_{J}^{(1)}(E) .
$$

Finally, substituting this result in Eq. (15) we get

$$
I_{J}(E)=I_{J}^{\text {direct }}(E)+I_{J}^{\text {central }}(E)+\frac{\gamma}{1-\gamma} I_{J}^{(1)}(E),
$$

where

$$
\gamma=\frac{2 \beta \alpha_{\infty}}{1-\alpha_{\infty}}=0.0294
$$

The contribution from all of the central gaps has been calculated separately using the scaling parameter $\beta_{0}=0.0795$ valid only for the central (nonasymptotic) part of the chattering region. This parameter scales the widths of the central gaps of successive generations,

$$
I_{J}^{\text {central }}(E)=\frac{\beta_{0}}{1-\beta_{0}} I_{J}^{(1)}(E) .
$$

Let us conclude this part by making the remark that some further approximations have been made in the derivation of Eq. (20). In particular, it has been assumed that the intergenerational scaling law is valid for all generations, and that the intragenerational scaling law holds for all icicles of the second and higher generations. However, it can be expected that the errors introduced in this way are very small since the value of the parameter and the individual probabilities for icicles on high-order generations are very small. Also, it is important to note that in the calculation of the intensity of each icicle it is very important to use the uniform semiclassical expression (7), since in the asymptotic region the contributing pair of trajectories are very close.

Using expression (20) we have calculated the diffraction intensities for the elastic scattering of ${ }^{4} \mathrm{He}$ atoms from the $\mathrm{Cu}(117)$ surface at incident angles of $45^{\circ}$ and $75^{\circ}$. The corresponding diffraction order functions were presented in Fig. 3 , where it can be observed that the chattering region for $45^{\circ}$ is much narrower than for $75^{\circ}$. Also these two irregular regions look qualitatively different. However, as we discussed in Sec. III the asymptotic shape of the fractal structure is the same for both incident angles, and we can use the same scaling parameters in both cases. Only the contributions of the direct part and the central icicles in the chattering regions will make the scattering intensities different.

In Table III the results corresponding to $\theta_{i}=45^{\circ}$ are presented. The semiclassical calculations have been done at three levels of approximation. In the second column we report the result of considering the contribution from the direct scattering and the classically forbidden transitions for $J=2$. In the third column the contribution from the interference terms with the classically allowed trajectories has been added. Finally, in the fourth column the contribution from the chaotic trajectories, computed using Eq. (20), has also been considered. The agreement with the close coupling and experimental results is rather good. Moreover, by comparing the different semiclassical results we can conclude that the interference terms are quite important in this case, and on the contrary the importance of the chattering region is very small. Also our calculations show that within the fractal region the first generation gives the largest contribution.

In Table IV the results corresponding to $\theta_{i}=75^{\circ}$ are presented. In this case only two semiclassical values have been computed. The first one corresponds to the contribution from the direct collisions plus the forbidden contributions for channels $J=-7,-8$, and -9 , and in the second one the contribution from the chattering region has been added. The agreement with the close-coupling results is globally acceptable (in this case the experimental results have not been included in the table since the in-plane unitarity is very poor). Here the contribution from the fractal is more important than in the previous case. In the last column of Table IV we have included the results which are obtained when the chattering region is treated using a statistical (Monte Carlo) method. ${ }^{9}$ In this case, the results obtained in this way are similar to those obtained by the present semiclassical method. At first sight this is very surprising since the chattering region is highly structured. We note that in the present conditions of energy and incident angle the system is close to a selective adsorption resonance condition, ${ }^{19}$ a phenomenon where quantummechanical tunneling is crucial. It is possible that the formalism should be modified to take into account classically forbidden processes inside the chattering region, a point which is currently being investigated. 
TABLE III. Diffraction intensities for the scattering of ${ }^{4} \mathrm{He}$ atoms from a $\mathrm{Cu}(117)$ surface at $21 \mathrm{meV}$ and $\theta_{i}=45^{\circ}$.

\begin{tabular}{|c|c|c|c|c|c|}
\hline $\begin{array}{l}\text { Diffraction } \\
\text { channel }\end{array}$ & $\begin{array}{l}\text { Semiclassical } \\
\text { (a) }\end{array}$ & $\begin{array}{l}\text { Semiclassical } \\
\text { (b) }\end{array}$ & $\begin{array}{l}\text { Semiclassical } \\
\text { (c) }\end{array}$ & Close-coupling & $\begin{array}{c}\text { Expt. } \\
\text { (Ref. 18) }\end{array}$ \\
\hline 2 & 0.2627 & 0.4637 & 0.464 & 0.464 & 0.781 \\
\hline 1 & 0.0209 & 0.0209 & 0.0234 & 0.0365 & \\
\hline 0 & 0.0387 & 0.0387 & 0.044 & 0.0313 & 0.010 \\
\hline-1 & 0.0916 & 0.0916 & 0.0975 & 0.0581 & 0.124 \\
\hline-2 & 0.0355 & 0.0355 & 0.0402 & 0.0155 & 0.019 \\
\hline-3 & 0.0077 & 0.0077 & 0.0107 & 0.0086 & \\
\hline-4 & 0.0402 & 0.0402 & 0.0402 & 0.0612 & 0.019 \\
\hline-5 & 0.0861 & 0.0861 & 0.0861 & 0.104 & 0.019 \\
\hline-6 & 0.0897 & 0.0897 & 0.0897 & 0.101 & \\
\hline-7 & 0.0623 & 0.0623 & 0.0623 & 0.0661 & \\
\hline$-8^{\mathrm{d}}$ & 0.032 & 0.032 & 0.032 & 0.033 & 0.025 \\
\hline$-9^{\mathrm{d}}$ & 0.012 & 0.012 & 0.012 & 0.02 & \\
\hline
\end{tabular}

${ }^{a}$ Results including the contribution from the direct scattering plus classically forbidden transitions for channels $J=2,-8$, and -9 .

${ }^{\mathrm{b}}$ Same as (a) but including the interference terms between classically allowed and classically forbidden trajectories, which are particularly important in this case.

${ }^{\mathrm{c}}$ Same as (b) but including the contribution from the chattering region [see Fig. 3(a)] calculated using Eq. (20). No interference cross terms between the direct and the chattering region were taken into account.

${ }^{\mathrm{d}}$ Classically forbidden transitions.

Finally, a number of questions still remains to be studied. Among them we can cite the role of the Maslov's index. In the present calculations we have set their differences to zero, assuming that the conclusions obtained for the regular case (Sec. II) also hold in the chaotic regime for each individual icicle. However, a more systematic study needs to be done.

\section{CONCLUSIONS}

Although the nature of atom-surface diffraction process is quantum mechanical in nature, we can use information of the underlying classical dynamics to correctly describe some of the associated observables, and even incorporate properly quantum effects (tunneling) when the contribution of classically forbidden trajectories is included. This was an important achievement of the classical $S$-matrix theory two decades ago. ${ }^{2}$ The semiclassical approach gives much of the physical insight lost when only the "correct" quantum treatment is used. However, the $S$-matrix theory encounters serious problems when the classical trajectories of the system are chaotic. The dynamics of atom-surface scattering can be very complex, and it is important to recognize how this com-

TABLE IV. Diffraction intensities for the scattering of ${ }^{4} \mathrm{He}$ atoms from a $\mathrm{Cu}(117)$ surface at $21 \mathrm{meV}$ and $\theta_{i}=75^{\circ}$.

\begin{tabular}{rlccc}
\hline \hline $\begin{array}{c}\text { Diffraction } \\
\text { channel }\end{array}$ & $\begin{array}{c}\text { Semiclassical } \\
\text { (a) }\end{array}$ & $\begin{array}{c}\text { Semiclassical } \\
\text { (b) }\end{array}$ & Close-coupling & $\begin{array}{c}\text { Monte Carlo } \\
\text { (c) }\end{array}$ \\
\hline 0 & 0.1149 & 0.212 & 0.0104 & 0.193 \\
-1 & 0.003 & 0.1285 & 0.0296 & 0.117 \\
-2 & 0.0373 & 0.110 & 0.149 & 0.1088 \\
-3 & 0.1031 & 0.136 & 0.232 & 0.1367 \\
-4 & 0.1265 & 0.198 & 0.23 & 0.1668 \\
-5 & 0.1049 & 0.1098 & 0.17 & 0.1265 \\
-6 & 0.0663 & 0.0679 & 0.101 & 0.0713 \\
$-7^{\mathrm{d}}$ & 0.034 & 0.034 & 0.0486 & \\
$-8^{\mathrm{d}}$ & 0.0198 & 0.0198 & 0.0199 & \\
$-9^{\mathrm{d}}$ & 0.0079 & 0.0079 & 0.0105 & \\
\hline \hline
\end{tabular}

${ }^{a}$ Results including the contribution from the direct scattering plus classically forbidden transitions for channels $J=-7,-8$, and -9 . No interference between classically allowed and classically forbidden trajectories need to be taken into account [see Fig. 3(b)].

${ }^{\mathrm{b}}$ Same as (a) but including the contribution from the chattering region.

${ }^{c}$ The contribution of the chattering region has been calculated here using a statistical (Monte Carlo) method, as proposed in Ref. 9.

${ }^{\mathrm{d} C}$ Classically forbidden transitions. 
plexity comes out. For a wide range of parameters (initial incident angle, total energy or the corrugation of the surface) the signatures of classical chaos can be found. In this case the onset of chaos is always related to a loss of enough momentum in the perpendicular mode, due to the corrugation of the surface, so that the atom cannot escape to the asymptotic region and gets trapped. ${ }^{17}$ The occurrence of fractal structures in chaotic scattering problems is now a well established fact, and a considerable amount of information has been provided in the past. ${ }^{8-17}$ Analogous features to those found in the present system, and the relation between the dynamics and the structure of the fractals is of universal nature and has been found in different systems, such as atom-molecule collisions. ${ }^{9-11,13,15,16}$

In this paper we have followed most of the procedures proposed in Ref. $13 \mathrm{~b}$ to extend, making use of the selfsimilarity of the fractal, the "standard" classical $S$-matrix theory, ${ }^{2}$ to chaotic atom-surface scattering problems. The agreement found between the semiclassical results obtained with this method and the quantum close-coupling results should stimulate the interest for carrying out similar studies for other elementary processes taking place in the scattering with surfaces, such as the existence of selective adsorption resonances, sticking, or inelastic and diffusive scattering. It is our opinion that within the theoretical formalism developed here that a very interesting view of those processes can be obtained. Also, we have found that the inclusion of the interference terms between classically allowed and classically forbidden processes is very important to get intensities which are unitary. In this paper the influence of the vibrations of the surface and its temperature on the diffraction has not been taken into account. We have started some work along this line, and preliminary results indicate that the trapping probability is highly enhanced when the surface vibrations, even at $0 \mathrm{~K}$, are considered.

\section{ACKNOWLEDGMENTS}

This work has been supported in part by DGICYT (Spain) under Contracts No. PB92-53 and PB92-181, and the NSF (USA) Grant No. RII-8922106. C.J. and R.G. gratefully acknowledge a sabbatical and doctoral grants, respectively, from the Ministerio de Educación y Ciencia (Spain).
*Permanent address: Department of Chemistry, West Virginia University, Morgantown, WV 26506-6045.

${ }^{1}$ G. Comsa, in Dynamics of Gas-Surface Interactions, edited by G. Benedek and U. Valbusa, Springer Series in Chemical Physics, Vol. 21 (Springer-Verlag, Berlin, 1982), p. 79.

${ }^{2}$ R. A. Marcus, J. Chem. Phys. 54, 3965 (1971); 57, 4903 (1972); 59, 5135 (1973); W. H. Miller, Adv. Chem. Phys. 25, 69 (1974); 30, 77 (1975), and references therein.

3 (a) J. D. Doll, Chem. Phys. 3, 257 (1974); J. Chem. Phys. 61, 95 (1974); (b) K. J. McCann and V. Celli, Surf. Sci. 61, 954 (1974).

${ }^{4}$ (a) R. I. Masel, R. P. Merril, and W. H. Miller, J. Chem. Phys. 64, 45 (1976); 65, 2690 (1976); (b) L. M. Hubbard and W. H. Miller, J. Chem. Phys. 78, 1801 (1983); 80, 5827 (1984).

${ }^{5}$ V. P. Maslov and M. V. Fedoriuk, Semi-Classical Approximation in Quantum Mechanics (Reidel, Boston, 1981); J. B. Delos, Adv. Chem. Phys. 65, 161 (1986); S. G. Creagh, J. M. Robbins, and R. G. Littlejohn, Phys. Rev. A 42, 1907 (1990); G. Campolieti and Paul Brumer, Phys. Rev. A 50, 997 (1994).

${ }^{6}$ (a) M. V. Berry, J. Phys. A 8, 566 (1975); (b) J. N. L. Connor, Mol. Phys. 31, 33 (1976); J. N. L. Connor and D. Farrelly, J. Chem. Phys. 75, 2831 (1976); Chem. Phys. Lett. 81, 306 (1981); J. B. Delos, J. Chem. Phys. 86, 425 (1987); J. H. Jensen, Phys. Rev. A 45, 1307 (1992); (c) T. Poston and I. Stewart, Catastrophe Theory and its Applications (Pitman, San Francisco, 1978).

${ }^{7}$ J. N. L. Connor and R. A. Marcus, J. Chem. Phys. 55, 5636 (1971); W. H. Miller, ibid. 54, 5386 (1971).

${ }^{8}$ For some reviews, see, G. Troll and U. Smilansky, in Chaos and Quantum Physics, Proceedings of the Les Houches Summer School, Session LII 1989, edited by M. J. Giannoni, A. Voros, and J. Zinn-Justin (Elsevier, Amsterdam, 1990); B. Eckardt, Physica D 33, 89 (1988).

${ }^{9}$ C. C. Rankin and W. H. Miller, J. Chem. Phys. 55, 3150 (1971).

${ }^{10}$ J. R. Stine and R. A. Marcus, Chem. Phys. Lett. 29, 575 (1974).

${ }^{11}$ B. B. Grayce, R. T. Skodje, and J. M. Hutson, J. Chem. Phys. 98, 3929 (1993).

${ }^{12}$ (a) P. Gaspard and S. A. Rice, J. Chem. Phys. 90, 2225 (1989);
90, 2255 (1989); (b) C. Jung and S. Pott, J. Phys. A 23, 3729 (1990).

${ }^{13}$ (a) A. Tiyapan and C. Jaffé, J. Chem. Phys. 99, 2765 (1993); (b) 101, 10393 (1994).

${ }^{14}$ S. Bleher, C. Grebogi, and E. Ott, Physica D 46, 87 (1990); M. Ding, C. Grebogi, E. Ott, and J. Yorke, Phys. Rev. A 42, 7025 (1990); R. Blümel and U. Smilanski, Physica D 36, 111 (1989); G. Troll and U. Smilanski, ibid. 35, 34 (1989); J. Phys. A 20, 5971 (1987); C. Jung, ibid. 19, 1345 (1986); C. Jung and H. J. Scholz, ibid. 20, 3607 (1987).

${ }^{15}$ L. Gottdiener, Mol. Phys. 29, 1585 (1975).

${ }^{16}$ D. W. Noid, S. K. Gray, and S. A. Rice, J. Chem. Phys. 84, 2649 (1986); S. Kumar, N. Sathyamurthy, and K. C. Bhalla, ibid. 98, 4680 (1993).

${ }^{17}$ (a) R. Guantes, F. Borondo, C. Jaffé, and S. Miret-Artés, Int. J. Quant. Chem. 52, 515 (1994); (b) F. Borondo, C. Jaffé, and S. Miret-Artés, Surf. Sci. 317, 211 (1994).

18 (a) D. Gorse, B. Salanon, F. Fabre, A. Kara, J. Perreau, G. Armand, and J. Lapujoulade, Surf. Sci. 47, 611 (1984); (b) J. Lapujoulade and Y. Lejay, Surf. Sci. 69, 354 (1977); J. Lapujoulade, Y. Lejay, and N. Papanicolau, ibid. 90, 133 (1979); J. Perreau and J. Lapujoulade, ibid. 122, 341 (1982).

${ }^{19}$ M. Hernández, S. Miret-Artés, P. Villarreal, and G. DelgadoBarrio, Surf. Sci. 274, 21 (1992); 290, L693 (1993).

${ }^{20} \mathrm{M}$. Abramowitz and I. A. Stegun, Handbook of Mathematical Functions (Dover, New York, 1972).

${ }^{21}$ W. H. Miller and T. F. George, J. Chem. Phys. 56, 5668 (1972); 56, 5722 (1972); 57, 2458 (1972).

${ }^{22}$ S. Wiggins, Chaotic Transport in Dynamical Systems (SpringerVerlag, New York, 1992); A. Tiyapan and C. Jaffé, J. Chem. Phys. 103, 5499 (1995).

${ }^{23}$ B. B. Mandelbrot, The Fractal Geometry of Nature (Freeman, New York, 1983); T. Tél, Z. Naturforsch. 43a, 1154 (1988).

${ }^{24}$ Y. T. Lau, J. M. Finn, and E. Ott, Phys. Rev. Lett. 66, 978 (1991).

${ }^{25}$ R. Guantes, C. Jaffé, F. Borondo, and S. Miret-Artés (unpublished). 